\title{
The Influence of Flow and Motion in MRI of Diffusion Using a Modified CE-FAST Sequence
}

\author{
Klaus-Dietmar Merboldt, Wolfgang Hänicke, Michael L. Gyngell, \\ JENS FRAHM, AND HARALD BRUHN
}

Max-Planck-Institut für biophysikalische Chemie, Postfach 2841, D-3400 Göttingen, Federal Republic of Germany

Received June 24. 1988: revised January 23. 1989

\begin{abstract}
Severe motion and flow artifacts are a problem in MRI of diffusion in vivo due to the application of strong magnetic field gradients. Here it is shown that image artifacts can be removed by using a modified fast-scan MRI sequence (CE-FAST) in conjunction with averaging of diffusion-weighted images. In phantom studies slow (coherent) flow $(<1$ $\mathrm{mm} \mathrm{s}^{-1}$ ) in the presence of strong diffusion gradients is shown to cause signal losses in diffusion-weighted images that depend on the relative orientations of the flow direction and the diffusion gradient. On the other hand, pulsatile motions of macroscopic dimensions (e.g., $1 \mathrm{~mm}, \mathrm{I} \mathrm{Hz}$, in-plane) lead to smearing and ghosting of signal intensities along the phase-encoding direction of the images. In both phantoms and rabbit brains in vivo motion artifacts were found to be reducible by averaging $8-16$ images. Unfortunately, the resulting image contrast no longer represents a "true" diffusion contrast but is affected by additional signal losses due to motion averaging. All experiments were performed on a 40-cm-bore 2.35-T Bruker Medspec system. @1989 ^eademic Press. Inc
\end{abstract}

\section{INTRODUCTION}

Differences between tissues as well as between normal and diseased states may be reflected in the dynamic properties of intra- and extracellular water. Microscopic transport of water molecules in vivo is characterized either by random processes (molecular self-diffusion) or by flow of blood in capillaries (microcirculation, perfusion ). Diffusion-type NMR techniques allow us to measure the mean positional displacement of a moving particle in a given period of time ("diffusion time"). Incoherent processes always give rise to signal attenuation, whereas signal losses due to coherent motions in principle may be recovered by means of appropriate "motioncompensating" gradient waveforms.

In general, the separation of "diffusion" and "perfusion" effects in vivo (1) is complicated by three major problems: First, capillary blood flow is both coherent and incoherent. Thus a separation of coherently and incoherently moving spins does not automatically result in "perfusion" and "diffusion" images, respectively. Moreover, the relative contributions of coherent and incoherent capillary blood flow also depend on the time scale. For example, the diffusion time may be chosen in such a way that incoherent motions dominate. Second, in the brain only $2 \%$ (white matter) to $5 \%$ (gray matter) of the total spins are involved in microcirculation. Thus, an ex- 
tremely good SNR is required to unambiguously detect the resulting signal attenuation. Third, and possibly the most difficult problem, the desired signal attenuation due to microscopic translational motions compete with signal losses due to unwanted macroscopic motions such as organ pulsations, respiratory motions, peristalsis, patient movements, or even dynamic instrumental instabilities. It is the purpose of this study to evaluate the influence of unwanted motions on diffusion MR images in vivo.

\section{METHODOLOGICAL ASPECTS}

A variety of approaches based on spin-echo or stimulated echo acquisition schemes have already been employed to generate diffusion weighting in MR images (2-5). These methods are hampered by image artifacts due to a spatial misregistration of moving or flowing spins in the presence of the necessarily strong diffusion gradients, and by long measuring times required for recording a series of diffusion-weighted images. For example, sufficient diffusion contrast in MRI requires a 50\% signal attenuation due to improperly rephased diffusing spins. For a diffusion coefficient of $10^{-5}$ $\mathrm{cm}^{2} \mathrm{~s}^{-1}$ this may be achieved with two gradient pulses of $30 \mathrm{~ms}$ duration and $10 \mathrm{mT}$ $\mathrm{m}^{-1}$ strength separated by a diffusion time of $100 \mathrm{~ms}$. Under such conditions, the normally small phase errors due to unavoidable macroscopic motions become considerably amplified and lead to unavoidable image artifacts.

In principle, flow- or motion-induced phase errors in MRI may be circumvented by motion-rephasing gradient waveforms (6). For diffusion imaging, however, this theoretically attractive approach fails because the incorporation of the complex motion-rephasing waveforms must be performed without further prolongation of the diffusion time. Longer diffusion times would result in an unacceptable reduction of the SNR due to relaxation losses. Thus, motion rephasing either requires even higher gradient strengths in conflict with the specifications of most whole-body MRI systems or causes a reduction of the effective diffusion gradient in conflict with the reliability of the diffusion imaging experiment. Preliminary trials with diffusion gradients having constant velocity rephasing waveforms confirmed this problem using a conventional $1.5-\mathrm{T}$ whole-body imager equipped with a $10 \mathrm{mT} \mathrm{m}^{-1}$ gradient system. Moreover, no significant improvement with respect to the reduction of image artifacts in human brain studies was observed. Attempts to reduce the influence of pulsatile motions by ECG-triggering result in rather long measuring times and are unable to take care of other (nonperiodic) motions causing "in scan" signal losses during the effective diffusion time.

This paper presents applications of a recently proposed fast-scan diffusion MRI technique $(7,8)$ to diffusion studies in the presence of slow flow and pulsatile motion parallel and perpendicular to the direction of the diffusion gradients. A schematic diagram of the radiofrequency (RF) and magnetic field gradient sequence used for MRI of molecular self-diffusion is depicted in Fig. 1. Two repetition cycles out of $n$ phase-encoding steps are shown. The method is based on the CE-FAST MRI sequence (9) which acquires the echo part of the steady-state free precession (SSFP) signal. For data acquisition the position of the echo preceding the RF pulses is shifted by $13 \mathrm{~ms}$ in advance by reversal of the read gradient. For diffusion imaging an addi- 


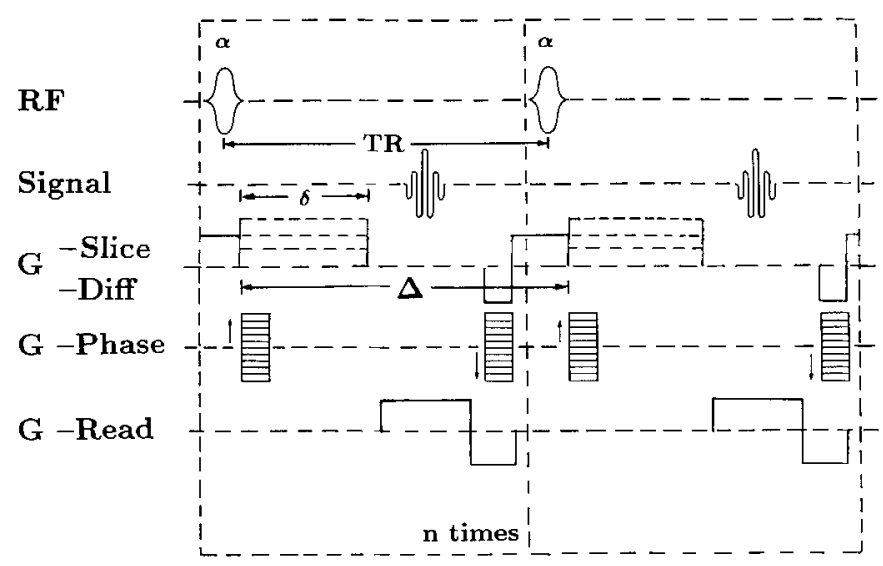

FIG. 1. Schematic diagram of the RF pulse and gradient sequence used for fast-scan MRI of diffusion. The method is based on the CE-FAST sequence modified to include a diffusion gradient G-DIFF of duration $\delta$ in addition to the conventional imaging gradients G-Slice, G-Phase, and G-Read. The diffusion gradient is balanced with respect to the acquired echo part of the SSFP signal. In this sequence the diffusion time $\Delta$ corresponds to the repetition time TR. Two repetition cycles out of $n$ are shown.

tional gradient which is balanced with respect to the acquired echo part of the SSFP signal is employed (compare the two subsequent cycles in Fig. 1). The diffusion time $\Delta$ for the primary spin echo of the SSFP signal is identical to the repetition time TR. However, for higher order echoes the diffusion times are correspondingly longer and therefore facilitate the generation of diffusion contrast for a given gradient strength.

The diffusion gradient may be applied in the direction of the read gradient $(7,8)$ or slice selection gradient (Fig. 1) or both. This variability may be exploited for the study of anisotropic diffusion or for the detection of directional differences of slow coherent flow. A simultaneous application of both gradients may be used for maximizing the available gradient power. In the present studies the maximum strength of a single diffusion gradient was limited to about $15 \mathrm{mT} \mathrm{m}^{-1}$. For the calculation of diffusion coefficients at least two images with different gradient strengths or durations are required. A simplified formula for the signal behavior in the diffusion-modified CE-FAST sequence has been reported elsewhere $(8)$. The treatment is based on a theoretical description of diffusion effects on spin-echo and stimulated echo contributions to the SSFP echo in the absence of motion.

It should be noted that motion-induced signal losses in SSFP imaging sequences are due to two distinct mechanisms. One is the "dephasing" of moving spins in the presence of a magnetic field gradient reflecting phase errors that accumulate between excitation and detection. Spatial integration of the effects may lead to partial or complete cancellation of signals within an image voxel. This type of signal loss is observed in any conventional non-SSFP sequence using gradient echoes, spin echoes, or stimulated echoes. The other mechanism is the breakdown of the steady state in an SSFP experiment due to unstable conditions. Crucial to an SSFP experiment is that the 
net phase accumulation per repetition cycle of a spin isochromat is constant. This parameter is a function of resonance offsets, repetition time, gradient amplitudes and waveforms, flow properties, and RF phase cycling.

\section{RESULTS AND DISCUSSION}

Macroscopic motions in diffusion experiments in vivo result in a misregistration of intensities in the phase-encoding direction of MR images. Accordingly, related signal losses in the images compete with the desired diffusion attenuation of a particular tissue. To verify the effects of flow and motion and to separate them from diffusion, phantom studies have been carried out before applying the developed strategies to diffusion imaging of rabbit brains in vivo.

\section{(i) The Influence of Slow Coherent Flow}

Experiments on slowly flowing spins were performed using a 5-cm (i.d.) tube with constant flow velocities controlled by regulation of the volume flow rate. Cross-sectional diffusion-weighted CE-FAST images were recorded with diffusion gradient pulses applied in the direction of either the slice selection gradient or the frequencyencoding ("read") gradient, i.e., parallel or perpendicular to the flow direction. Figure 2 shows transaxial images of the water phantom at zero flow (a), and at flow velocities of about $0.2 \mathrm{~mm} \mathrm{~s}^{-1}(\mathrm{~b}, \mathrm{c})$ and $0.6 \mathrm{~m} \mathrm{~m} \mathrm{~s}^{-1}(\mathrm{~d})$, respectively, in the presence of a diffusion gradient of $10 \mathrm{mT} \mathrm{m}^{-1}$. Images (a) and (b) refer to a repetition time (diffusion time) of $45 \mathrm{~ms}$ and a diffusion gradient of duration $15 \mathrm{~ms}$ perpendicular to the flow direction (G-Diff = G-Read), while images (c) and (d) were recorded using a repetition time of only $35 \mathrm{~ms}$ and a diffusion gradient of $5 \mathrm{~ms}$ parallel to the flow direction (G-Diff = G-Slice). Even though the diffusion-weighted CE-FAST images in Fig. 2 represent single-excitation images without data averaging nearly no phase-encoding artifacts are observed for coherent flow with velocities in the range of capillary flow in vivo. This is due to a complete dephasing of flowing spins in the presence of gradients that are not compensated for (constant) flow. In addition, flowing or moving spins may not establish a steady state of transverse coherences in a repetitive RF pulse experiment. Therefore, flowing/moving spins are best visualized using the FID in a FLASH-type experiment $(10)$, but normally do not give rise to SSFP echoes acquired in a CE-FAST sequence. This sensitivity of SSFP imaging sequences to slow flow is in agreement with previous findings by others (11).

The evaluated diffusion coefficient for stationary water is too high by a factor of about 3 (Fig. $2 \mathrm{a}$ ) if solely the primary spin-echo component of the SSFP echo signal is taken into account. Calculations have been performed on the basis of five images recorded with different gradient strengths (not shown). The deviation of the selfdiffusion coefficient of water is explained by the neglect of higher order echoes although significant contributions are expected for long spin-spin relaxation times in tap water and short repetition times as used here $(8,12-14)$. Similar findings were obtained in the presence of slow flow provided the diffusion gradients were oriented perpendicular to the flow direction (Fig. 2b). However, even for extremely small flow velocities of about $0.2 \mathrm{~mm} \mathrm{~s}^{-1}$ the effective diffusion coefficient increased by factors 

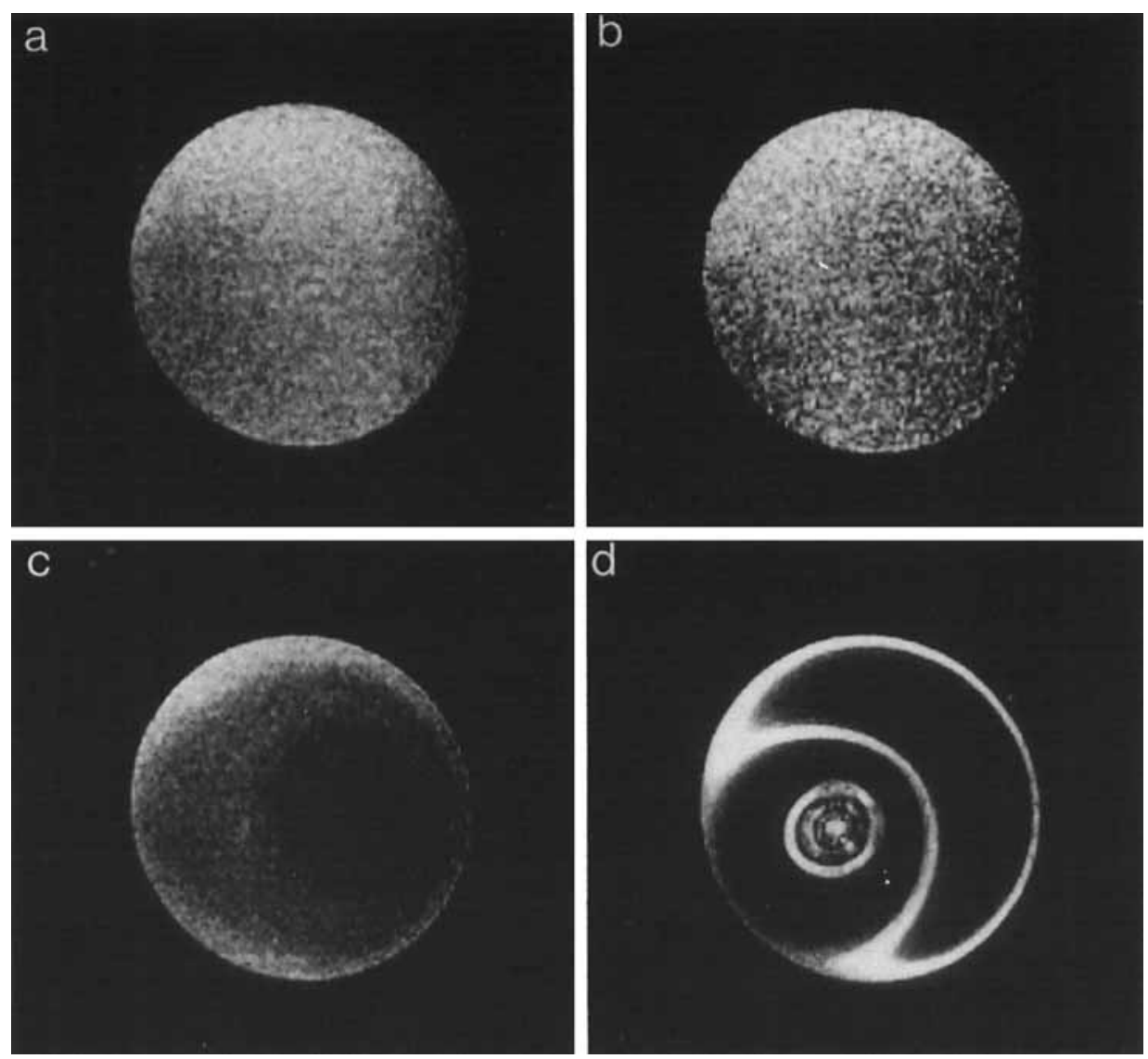

FIG. 2. The influence of slow flow in fast-scan MRI of diffusion. Transaxial diffusion-weighted CE-FAST images ( $256 \times 256$ pixel resolution, 12-cm FOV, 4-mm slice thickness, $90^{\circ}$ flip angle) of a 5-cm-diameter tube of water at zero flow (a) and at flow velocities of about $0.2 \mathrm{~mm} \mathrm{~s} \mathrm{~s}^{-1}$ (b,c) and $0.6 \mathrm{~mm} \mathrm{~s}^{-1}$ (d), respectively. Images (a) and (b) were recorded within a measuring time of $19 \mathrm{~s}$ using a repetition time (diffusion time) of $45 \mathrm{~ms}$ and a $10 \mathrm{mT} \mathrm{m}^{-1}$ diffusion gradient of $15 \mathrm{~ms}$ duration perpendicular to the flow direction (G-Diff $=\mathrm{G}$-Read). Images $(\mathrm{c})$ and $(\mathrm{d})$ were obtained within a measuring time of $15 \mathrm{~s}$ using a repetition time of $35 \mathrm{~ms}$ and a $10 \mathrm{mT} \mathrm{m}^{-1}$ diffusion gradient of $5 \mathrm{~ms}$ duration parallel to the flow direction (G-Diff = G-Slice) .

of up to 20 for a gradient oriented parallel to the flow direction (Fig. 2c). Moreover, the values differ across the transaxial image reflecting the existence of a velocity profile across the phantom with high values in the center of the tube and lower values near the boundary. If the flow velocity is increased a circular intensity pattern that may be explained by flow rephasing effects that occur for selected flow velocities in the approximately laminar flow profile (low Reynolds number) is observed.

\section{(ii) The Influence of Pulsatile Motion}

Pulsatile motions in fast-scan MRI of diffusion have been investigated using a periodic spatial displacement of the sample in the imaging plane with an amplitude of 
about $1 \mathrm{~mm}$ and a frequency of $1 \mathrm{~Hz}$. Diffusion imaging experiments were then carried out on a phantom consisting of three tubes of $\mathrm{Cu}\left(\mathrm{SO}_{4}\right)_{2}$-doped water, dimethyl sulfoxide (DMSO), and an aqueous solution of polyethylene glycol (PEG), respectively. Their diffusion coefficients were determined to be 2.1 for water, 1.2 for DMSO, and 0.5 for PEG in units of $10^{-5} \mathrm{~cm}^{2} \mathrm{~s}^{-1}$ using the conventional Stejskal-Tanner spin-echo technique (1.5). They cover the range of diffusion coefficients accessible in vivo. Series of diffusion-weighted fast-scan images were recorded with a repetition time of $60 \mathrm{~ms}$ and diffusion gradients of $30 \mathrm{~ms}$ duration with a strength ranging from 0 to $15 \mathrm{mT} \mathrm{m}$ '. Image artifacts are observed, in particular, if the direction of the motion coincides with the direction of the diffusion gradient. A considerable reduction of motion artifacts and the reestablishment of homogeneous image intensities were achieved by averaging the complex raw data of up to 16 sequentially recorded images as shown in Fig. 3.

Figure 4 depicts region of interest ( $\mathrm{ROI}$ ) intensities of $5 \times 5 \mathrm{~mm}^{2}$ areas taken from a series of images ( 16 acquisitions) recorded in the presence (open symbols) and absence (solid symbols) of motion. The half-logarithmic plot of normalized intensities $I(g)$ versus the square of diffusion gradient strength $g$ demonstrates the influence of motion on the diffusion measurement. In the presence of motion some intensity is lost due to the averaging of misregistered signals in the phase-encoding direction of the diffusion-weighted images. Since this intensity loss increases with diffusion gradient strength, the effective signal attenuation in the presence of motion is more pronounced than for pure diffusion. Of course, the extent of motion-related signal losses depends on the actual motion characteristics. Since the curves in Fig. 4 still approximate linear relationships the present experimental conditions would yield incorrect diffusion coefficients that are $25-100 \%$ higher than in the absence of motion. Small diffusion coefficients are affected more than higher ones, since the relative signal loss due to motion becomes more important in cases where slowly diffusing substances yield only small signal attenuations due to diffusion.

These experiments clearly indicate that the presence of pulsatile or nonperiodic motions as found in vivo severely complicates or prevents quantitative imaging of diffusion. The resulting contrasts in diffusion-weighted CE-FAST images represent a combination of true-diffusion contrasts and normally unknown signal losses due to the averaging of motion artifacts. It should be noted that the sequential recording of images and subsequent averaging of the raw data sets turned out to be superior to averaging of immediately repeated recordings of each individual phase-encoding step. This is because artificial coherences or pscudo-gating (16) effects are more easily avoided on a time scale given by the measuring time of an image rather than of an individual projection.

\section{(iii) Rabbit Brain Studies In Vivo}

In vivo diffusion MRI experiments using the modified CE-FAST sequence are shown in Fig. 5. Three transaxial (a-c) and sagittal (d-f) images are selected out of two series of five images with diffusion gradient strengths of $0 \mathrm{mT} \mathrm{m}^{-1}(\mathrm{a}, \mathrm{d}), 7.5 \mathrm{mT}$ 

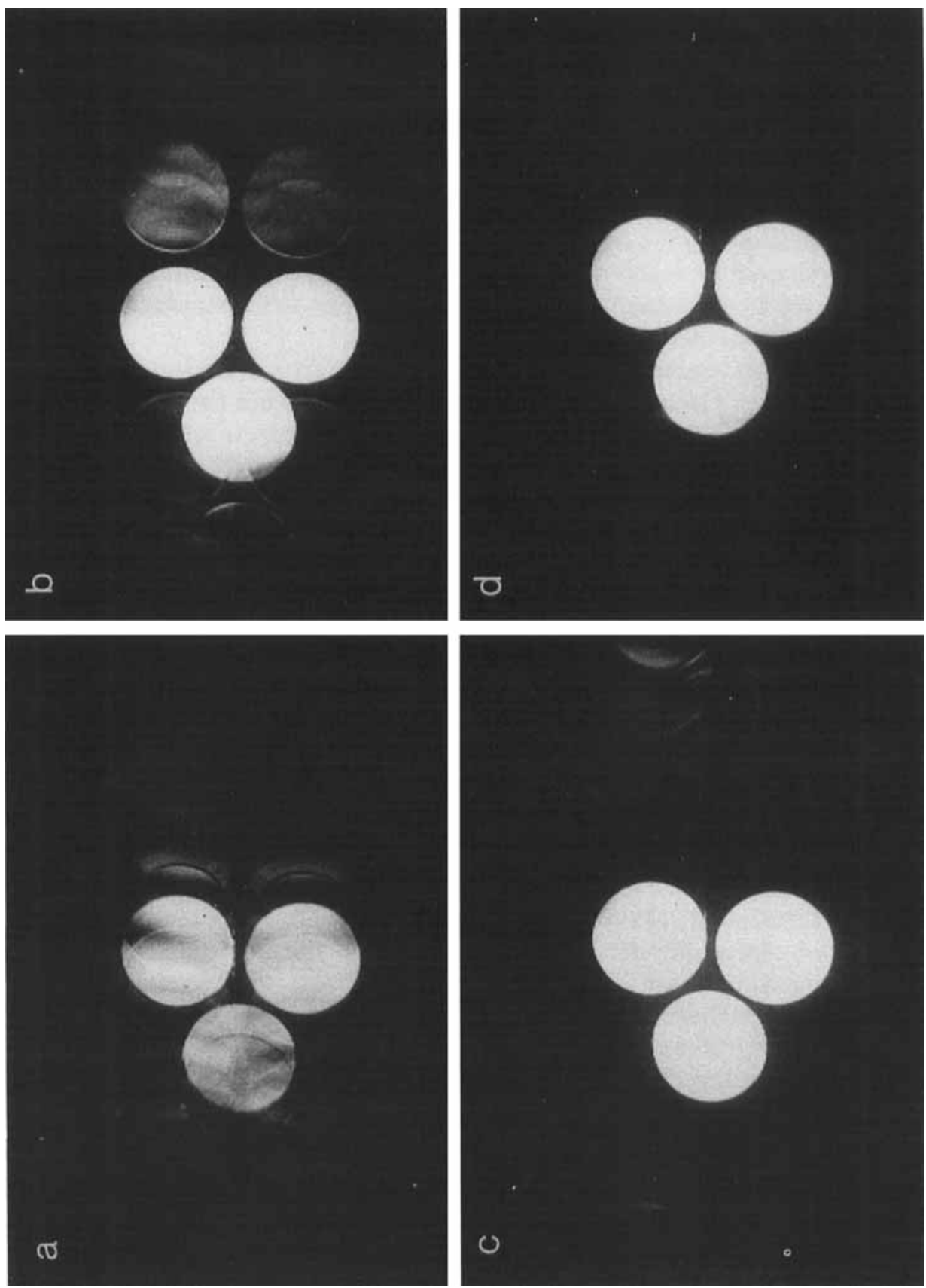


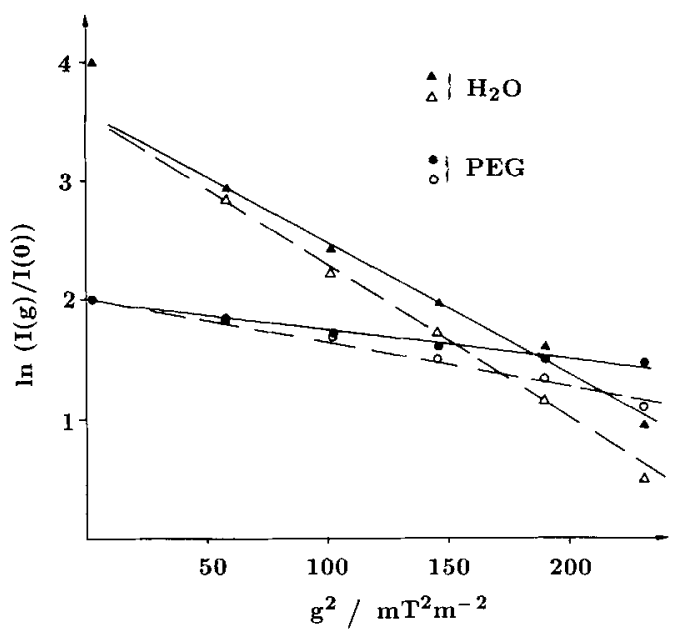

FIG. 4. The influence of small pulsatile motions of the sample ( $1 \mathrm{~mm}, 1 \mathrm{~Hz}$, in-plane) in fast-scan MRI of diffusion. Region of interest (ROI) values of $5 \times 5-\mathrm{mm}^{2}$ areas from series of diffusion-weighted CEFAST images recorded in the presence (open symbols) and absence (solid symbols) of motion are shown in a half-logarithmic plot of normalized intensities $I(g)$ versus the square of the diffusion gradient strength $g$. The data represent averages of 16 acquisitions with a repetition time of $60 \mathrm{~ms}$ and diffusion gradients of $30 \mathrm{~ms}$ duration. The gradient strengths varied from 0 to $15 \mathrm{~m} \mathrm{~T} \mathrm{~m}^{-1}$ in the direction of the read gradient.

$\mathrm{m}^{-1}(\mathrm{~b}, \mathrm{e})$, and $13.2 \mathrm{mT} \mathrm{m}^{-1}(\mathrm{c}, \mathrm{f})$, respectively. The images were recorded with a repetition time of $45 \mathrm{~ms}$ and a diffusion gradient of $15 \mathrm{~ms}$ duration. Reduction of motion artifacts was accomplished by averaging eight acquisitions resulting in a measuring time of about $1.5 \mathrm{~min}$ for each image with $256 \times 256$ pixel resolution.

In accordance with the preceding flow studies the signal intensity of the CSF is already lost in images obtained with rather small diffusion gradients (Figs. 5b and 5e). A change of the orientation of the diffusion gradient from the slice selection direction to the frequency-encoding direction did not cause any significant differences. This finding indicates the absence of a preferential flow direction supported by the relatively large voxel sizes obtained using a 4-mm slice thickness and the tiny vascular structures in the rabbit brain. A further increase in the gradient strength (Figs. $5 \mathrm{c}$ and $5 \mathrm{f}$ ) results in a signal attenuation of brain tissue that may be ascribed to diffusional processes and possible contributions from averaged artifacts due to brain pulsations. As expected the signal from subcutaneous fat remains nearly unaffected due to the considerably lower diffusion coefficients of lipids.

Figure 6 shows signal intensities from the images in Fig. 5 as a function of the square of the diffusion gradient strength $g$. The data refer to normalized intensities $I(g)$ of ROI values for CSF $\left(2 \times 2 \mathrm{~mm}^{2}\right)$, brain tissue $\left(5 \times 5 \mathrm{~mm}^{2}\right)$, and fat $(5 \times 5$

FIG. 3. The influence of small pulsatile motions of the sample ( $1 \mathrm{~mm}, 1 \mathrm{~Hz}$, in-plane $)$ in fast-scan MRI of diffusion. Transaxial CE-FAST images ( $256 \times 256$ pixel resolution, 12-cm FOV, 4-mm slice thickness, $90^{\circ}$ flip angle) of three vials containing water, DMSO, and PEG. The images represent averages of 1 (a), 4 (b), $8(\mathrm{c})$, and 16 (d) acquisitions using a repetition time of $60 \mathrm{~ms}$. 

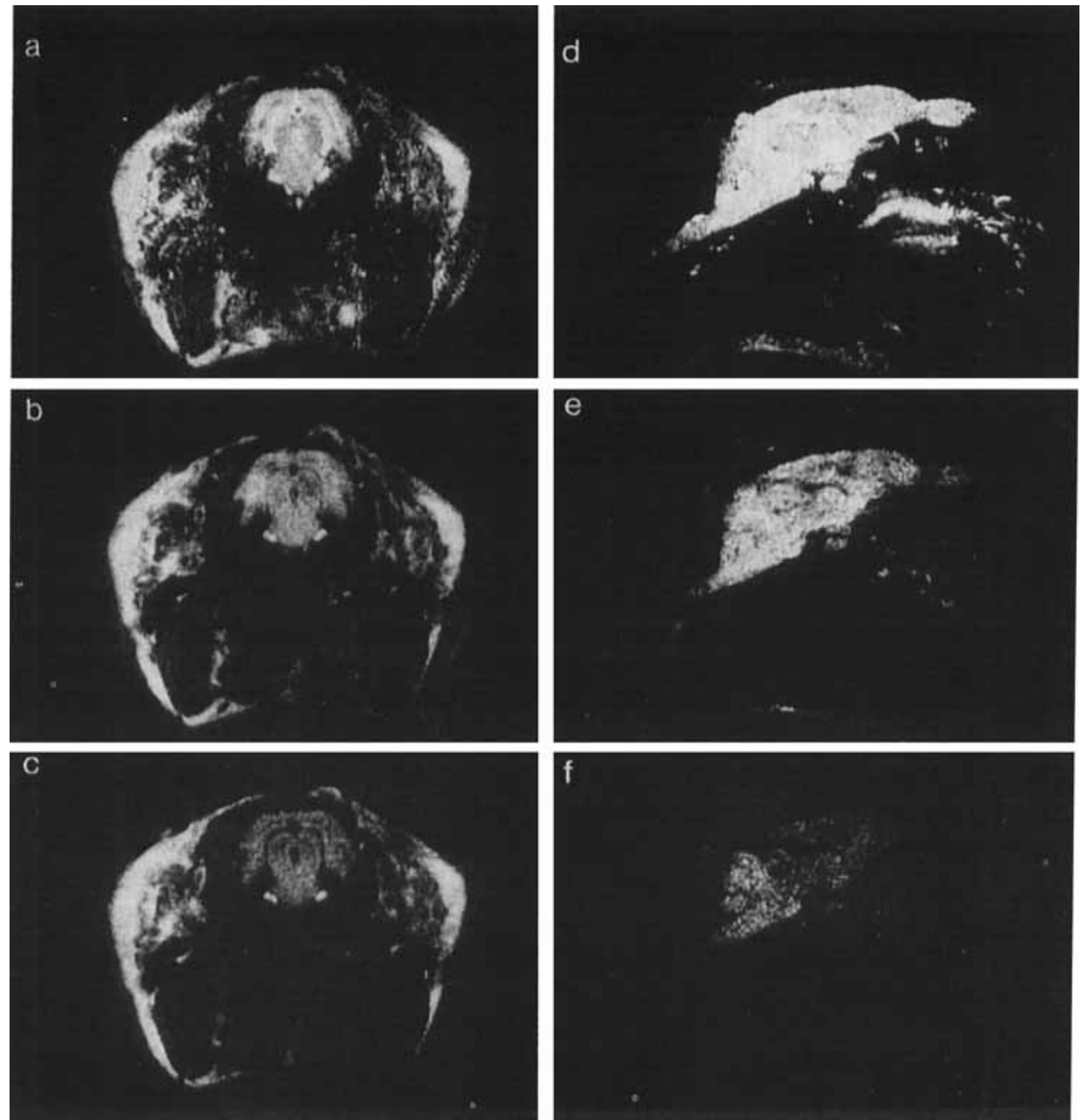

FIG. 5. Fast-scan diffusion MRI of a rabbit brain in vivo. Three transaxial ( $a-c)$ and sagittal $(\mathrm{d}-\mathrm{f})$ images $\left(256 \times 256\right.$ pixel resolution, $12-\mathrm{cm}$ FOV, 4-mm slice thickness, $90^{\circ}$ flip angle) are selected out of a series of five diffusion-weighted CE-FAST images with gradient strengths of $0 \mathrm{mT} \mathrm{m}^{\prime}$ (a. d) and $7.5 \mathrm{mT} \mathrm{m}^{-1}$ (b, e) to $13.2 \mathrm{mT} \mathrm{m}$ ' (c, f), respectively. The images represent averages of eight acquisitions resulting in a measuring time of about $1.5 \mathrm{~min}$. They were recorded with a repetition time of $45 \mathrm{~ms}$ and a diffusion gradient of $15 \mathrm{~ms}$ duration. The images obtained with a medium gradient strength exhibit a complete loss of CSF signals caused by noncompensated flow, while the images with the highest gradients show a signal attenuation of brain tissue due to diffusional processes.

$\mathrm{mm}^{2}$ ). In the half-logarithmic plot, the slopes directly correspond to different effective diffusion coefficicnts including contributions from macroscopic motions. Since diffusion effects in fat tissues may be neglected under the present experimental conditions the slight decrease in the lipid signal with increasing diffusion gradient represent a measure of signal losses due to unavoidable motions of the animal or other instru- 


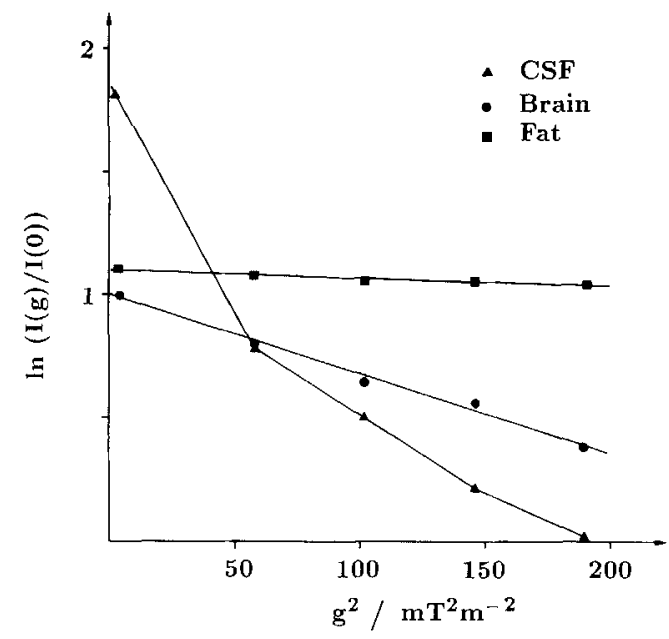

FIG. 6. Half-logarithmic plot of normalized intensities $I(g)$ from selected ROI of the brain images shown in Fig. 5 as a function of the square of the diffusion gradient strength $g$. ROI values are taken from areas corresponding to CSF ( $2 \times 2 \mathrm{~mm}^{2}$, triangles), brain tissue $\left(5 \times 5 \mathrm{~mm}^{2}\right.$, circles), and fat ( $5 \times 5 \mathrm{~mm}^{2}$, squares). The slopes directly correspond to different effective diffusion coefficients. Since lipids are known to exhibit very low diffusion coefficients their signals provide a simple measure for the extent of signal attenuation due to macroscopic motions.

mental instabilities. Even though the effective diffusion coefficient for rabbit brain tissue may be increased due to the presence of motion, its value is only about $60 \%$ of the value obtained for stationary water under similar conditions (compare Fig. 2a). This is in agreement with the occurrence of restricted diffusion in biological systems $(17,18)$. Finally, CSF signals exhibit a considerably higher effective diffusion coefficient caused by noncompensated flow effects.

\section{CONCLUSIONS}

The effects of slow coherent flow and pulsatile motions on diffusion-weighted CEFAST MR images have been investigated in phantoms. In vivo studies have been performed on rabbit brains. Phase-encoding image artifacts due to macroscopic motions are shown to be reduced by averaging of a moderate number of fast scan images. Thus, imaging times for individual diffusion-weighted images have been kept to $1-2$ min. Theoretical treatments and quantitative determinations of molecular self-diffusion coefficients are precluded because of both the complex behavior of the SSFP echo signal and the unpredictable signal losses due to macroscopic motions. Further complications may arise from distorted slice profiles. In fortunate cases, qualitative diffusion contrasts may be maintained after elimination of motion artifacts by averaging. This is confirmed by initial applications of diffusion-weighted CE-FAST images to studies of the normal and pathological human brain (19).

\section{ACKNOWLEDGMENT}

Financial support by the Bundesminister für Forschung und Technologie (BMFT) of the Federal Republic of Germany (Grant 01 VF 8606/6) is gratefully acknowledged. 


\section{REFERENCES}

1. D. Le Bihan, E. Breton, D. Lallemand, M. L. Aubin, J. Vignaud, and M. Laval-Jeantet, Radiology 168, 497 (1988).

2. D. G. TAylor and M. C. Bushell, Phys. Med. Biol. 30, 345 (1985).

3. K. D. Merboldt, W. Hänicke, AND J. Frahm, J. Magn. Reson. 64, 479 (1985).

4. D. Le Bihan, E. Breton, D. Lallemand, P. Grenier, E. Cabanis, and M. Laval-Jeantet, Radi-

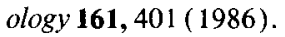

5. K. D. Merboldt, W. Hänicke, and J. Frahm, Ber. Bunsenges. Phys. Chem. 91, 1124 (1987).

6. P. M. Pattany, J. J. Phillips, L. C. Chiu, J. L. Duerk, J. M. McNally, and S. N. Mohapatra, J. Comput. Assist. Tomogr. 11, 369 (1987).

7. D. LE BiHaN, Magn. Reson. Med. 7, 346 (1988).

8. K. D. Merboldt, W. Hänicke, M. L. Gyngell, J. Frahm, and H. Bruhn, J. Magn. Reson. 82, 115 (1989).

9. M. L. GYNGELL, Magn. Reson. Imaging 6, 415 (1988).

10. J. Frahm, A. HaAse, and D. MatthaEl, Magn. Reson. Med. 3, 321 (1986).

11. S. PATZ AND R. C. HAWkeS, Magn. Reson. Med. 3, 140 (1986).

12. D. E. Woessner, J. Chem. Phys. 34, 2057 (1961).

13. R. Kaiser, E. Bartholdi, AND R. R. Ernst, J. Chem. Phys. 60, 2966 (1974).

14. M. L. GYNGELL, J. Magn. Reson. 81, 474 (1989).

15. E. O. Stejsk AL AND J. E. TANner, J. Chem. Phys. 42, 288 (1965).

16. E. M. HAACKe, G. W. LeNZ, AND A. D. Nelson, Magn. Reson. Med. 4, 162 (1987).

17. G. G. Cleveland, D. C. Chang, C. F. Hazlewood, and H. V. Rorschach, Biophys. J. 16, 1043 (1976).

18. J. E. TANNER, Biophys. J. 28, 107 (1979).

19. K. D. Merboldt, H. Bruhn, J. Frahm, M. L. Gyngell, W. HäniCKe, and M. Deimling, Magn. Reson. Med. 9, 423 (1989). 